\title{
Preparation and Electronic Property Investigation of Zinc(II)-Schiff Base Complexes in the Confined Space
}

\author{
Peng Shang' and Lei Zhang ${ }^{2}$ \\ ${ }^{1}$ School of Chemistry, Nankai University, Tianjin 300071, China \\ ${ }^{2}$ Institute of Modern Optics and MOE Key Laboratory of Optical Information Science \& Technology, Nankai University, \\ Tianjin 300071, China
}

Correspondence should be addressed to Lei Zhang; lzzhang@nankai.edu.cn

Received 10 March 2013; Revised 5 May 2013; Accepted 8 May 2013

Academic Editor: Theocharis C. Stamatatos

Copyright (c) 2013 P. Shang and L. Zhang. This is an open access article distributed under the Creative Commons Attribution License, which permits unrestricted use, distribution, and reproduction in any medium, provided the original work is properly cited.

\begin{abstract}
Metal-Schiff base complexes have attracted continued research interest regarding their intriguing and useful features, while the electronic properties of these complexes in the confined space have not been sufficiently addressed in previous studies. In this work, a new zinc(II)-Schiff base complex bis( $N$-dodecyl salicylideneiminato) $\mathrm{Zn}$ (II) (1) was synthesized and subsequently loaded in an inorganic solid host. A large red shift $(\sim 40 \mathrm{~nm})$ of the absorption onset was recorded, when the microenvironment of 1 changed from the solvent ethanol to the inorganic solid medium, evidencing the confined space effect. The marked shift of the absorption onset was associated with a band-gap reduction between the highest occupied molecular orbital (HOMO) and the lowest unoccupied molecular orbital (LUMO). Theoretical calculation results showed that the confined space effect is distance dependent and exerts a more profound influence on the HOMO than the LUMO within an effective distance range. An initial study implied that the confined space effect is also accompanied with the electron density variation.
\end{abstract}

\section{Introduction}

Schiff bases, named after Hugo Schiff, have gained immense popularity in the chemical community as a result of their attractive properties and diverse applicability [1-5]. Electronic, magnetic, optical, chemical, and biological properties of numerous Schiff bases and their metal complexes have been recently reported [6-10]. When an organic molecule is placed as a guest inside a host material, its physical and chemical properties are subject to changes, among others due to the so-called "confined space effect" [11]. Pioneered by the work on loading the conducting polyaniline filaments in a mesoporous channel host [12], organic molecules in the confined space have attracted considerable research interest [13-15]. Kitayama and colleagues experimentally demonstrated the beneficial use of the confined space effect in the nitroxidemediated radical microemulsion polymerization [16]. It is also discovered that combining the merits of the confined space effect and the synergic effect represents a promising way to construct versatile and efficient catalytic systems [17].
Metal-Schiff base complexes confined in a host material is an area that deserves to receive more research attention. It may be noted that various attempts have been made to prepare these composite systems with an emphasis of investigating their physical and chemical properties [18-20]. However, the change of the electronic properties of metalSchiff base complexes in the confined space has not been sufficiently addressed in the previous studies. Therefore, in this contribution, we synthesize a zinc(II)-Schiff base complex, load it in an inorganic solid host, and carry out experimental and theoretical investigations toward a better understanding of its electronic properties.

\section{Materials and Methods}

2.1. Sample Preparation. All the chemicals used in this work were of analytical reagent grade and used without further purification, unless otherwise is stated. A new zinc(II)-Schiff base complex, bis( $N$-dodecyl salicylideneiminato)Zn(II) (1), 
TABLE 1: Crystal data and structure refinement for $\mathbf{1 .}$

\begin{tabular}{lc}
\hline Empirical formula & $\mathrm{C}_{19} \mathrm{H}_{30} \mathrm{NOZn}_{0.5}$ \\
Formula weight & 321.14 \\
Crystal system & Monoclinic \\
Space group & $C 2 / c$ \\
$a(\AA)$ & $20.779(4)$ \\
$b(\AA)$ & $5.4540(10)$ \\
$c(\AA)$ & $32.660(5)$ \\
$\alpha\left(^{\circ}\right)$ & 90.00 \\
$\beta\left(^{\circ}\right)$ & $100.555(13)$ \\
$\gamma\left(^{\circ}\right)$ & 90.00 \\
$V\left(\AA^{3}\right)$ & $3638.7(11)$ \\
$Z$ & 8 \\
$D_{c}\left(\mathrm{~g} \mathrm{~cm}^{-3}\right)$ & 1.172 \\
$F_{000}$ & 1392 \\
$\mu\left(\mathrm{mm}^{-1}\right)$ & 0.708 \\
$\theta$ range for collection $\left(^{\circ}\right)$ & $2.93-25.01$ \\
No. of reflections collected & 7239 \\
No. of unique reflections & 3220 \\
$R_{\text {int }}$ & 0.0505 \\
No. of observed reflections $(I>2 \sigma(I))$ & 2566 \\
Parameters & 196 \\
$R_{1}(I>2 \sigma(I))$ & 0.0465 \\
$w R_{2}(I>2 \sigma(I))$ & 0.0756 \\
$R_{1}($ all data) & 0.0638 \\
$w R_{2}($ all data $)$ & 0.0837 \\
$\mathrm{GOF}$ & 1.179 \\
\hline & \\
\hline &
\end{tabular}

was synthesized in this work by mixing salicylaldehyde $(0.244 \mathrm{~g}, 2.0 \mathrm{mmol})$, dodecylamine $(0.371 \mathrm{~g}, 2.0 \mathrm{mmol})$, and $\mathrm{Zn}\left(\mathrm{CH}_{3} \mathrm{COO}\right)_{2} \cdot 2 \mathrm{H}_{2} \mathrm{O}(0.219 \mathrm{~g}, 1.0 \mathrm{mmol})$ in an ethanol solution $(30 \mathrm{~mL})$ at $70^{\circ} \mathrm{C}$ for $30 \mathrm{~min}$. The filtered solution was allowed to slowly evaporate at room temperature for several days to yield faint yellow crystals of $\mathbf{1}$ suitable for X-ray diffraction studies. Anal. Calcd for $\mathrm{C}_{19} \mathrm{H}_{30} \mathrm{NOZn}_{0.5}$ : C, 71.06; H, 9.42; N, 4.36. Found: C, 70.57; H, 9.45; N, 4.31.

The MCM-41-type mesoporous material as the solid host was synthesized and characterized according to the literature [21], revealing a $3.0 \mathrm{~nm}$ wide effective mean pore diameter. To load 1 into this solid host, $1(0.016 \mathrm{~g}, 0.05 \mathrm{mmol})$ was dissolved in a $\mathrm{CH}_{2} \mathrm{Cl}_{2}$ solution $(100 \mathrm{~mL})$, which was followed by the addition of the mesoporous material $(1.0 \mathrm{~g})$. The above reaction mixture was stirred for $4 \mathrm{~h}$ at room temperature. A light yellow powder was isolated by filtration and then washed copiously with $\mathrm{CH}_{2} \mathrm{Cl}_{2}$ solution.

2.2. Single-Crystal X-Ray Crystallography. The X-ray diffraction data of 1 were collected at $293 \pm 2 \mathrm{~K}$ on Oxford Diffractometer SuperNova with a graphite-monochromated Mo K $\alpha$ radiation $(\lambda=0.71073 \AA)$ using the $\omega$-scan technique. The structure was solved by direct methods and refined by fullmatrix least-squares technique on $F^{2}$ with anisotropic displacement parameters for non-H atoms using the SHELXS-97 and SHELXL-97 programs. The hydrogen atoms were placed
TABLE 2: Selected bond lengths $(\AA)$ and angles $\left(^{\circ}\right)$ for $\mathbf{1}$.

\begin{tabular}{lccc}
\hline Bond & $(\AA)$ & Angle & $\left(^{\circ}\right)$ \\
\hline Zn1-O1 & $1.9185(17)$ & O1-Zn1-N1 & $96.50(8)$ \\
Zn1-N1 & $1.991(2)$ & O1A-Zn1-N1A & $96.51(8)$ \\
Zn1-O1A & $1.9185(17)$ & O1-Zn1-N1A & $120.96(8)$ \\
Zn1-N1A & $1.991(2)$ & O1A-Zn1-N1 & $120.96(8)$ \\
N1-C7 & $1.291(3)$ & O1-Zn1-O1A & $110.93(11)$ \\
N1A-C7A & $1.291(3)$ & N1-Zn1-N1A & $112.77(12)$ \\
\hline
\end{tabular}

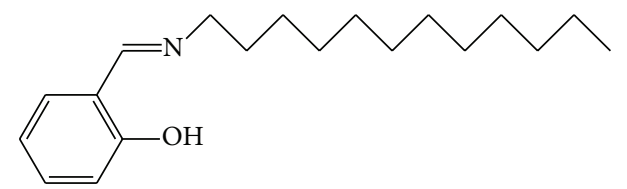

FIGURE 1: Chemical structure of the Schiff base ligand.

in idealized positions and constrained to ride on their parent atoms. A summary of the key crystallographic information is given in Table 1. Selected bond lengths and angles are given in Table 2. Detailed information regarding the crystallographic data can be retrieved from CCDC (deposition number: 920893).

\section{Results and Discussion}

A Schiff base ligand bearing a long alkane chain is used in this work. Figure 1 depicts the chemical structure of this ligand. The molecular structure of $\mathbf{1}$ was determined by single-crystal X-ray crystallography. 1 crystallizes in the monoclinic space group $C 2 / c$ with eight molecules in a unit cell. Figure 2 reveals a V-shaped molecular structure of 1 . The $\mathrm{Zn}$ (II) ion is four-coordinated, and the coordination sphere of the $\mathrm{Zn}$ (II) ion is best described as a distorted tetrahedral geometry completed by two oxygen atoms and two nitrogen atoms from the Schiff base ligands. 1 belongs to a $C 2$ point group, where the $C 2$ axis passes through the $\mathrm{Zn}$ (II) ion and the midpoint of two nitrogen atoms. Huo and colleagues recently synthesized $\mathrm{Zn}$ (salicylidene- $p$-methylaniline) ${ }_{2}$ (2), a zinc(II)-Schiff base complex that also crystallizes in the monoclinic space group $\mathrm{C} 2 / \mathrm{c}$ [22]. The $\mathrm{Zn}-\mathrm{O}$ bond length of $1(1.9185(17) \AA)$ is almost the same as that of 2 (1.9113(15) $\AA)$, while the $\mathrm{Zn}-\mathrm{N}$ bond length of $\mathbf{1}$ (1.991(2) $\AA$ ) is very close to that of $2(2.0020(16) \AA)$. The bond angles of $\mathrm{O}-\mathrm{Zn}-\mathrm{O}$ $\left(110.93(11)^{\circ}\right)$ and $\mathrm{N}-\mathrm{Zn}-\mathrm{N}\left(112.77(12)^{\circ}\right)$ of 1 are smaller than those of $\mathrm{O}-\mathrm{Zn}-\mathrm{O}\left(120.85(11)^{\circ}\right)$ and $\mathrm{N}-\mathrm{Zn}-\mathrm{N}\left(124.11(10)^{\circ}\right)$ of 2. This noticeable bond angle variation is most likely due to the structural difference of the Schiff base ligands in these two compounds.

The loading of $\mathbf{1}$ in the solid host was analyzed by powder X-ray diffraction (PXRD). Figure 3 shows the PXRD patterns of pure MCM-41 and MCM-41 loaded with 1. The PXRD patterns of both materials show Bragg peaks at low reflection angles, which are typical of the mesoporous MCM-41. The PXRD pattern of MCM-41 loaded with 1 is generally identical to that of the pure MCM-41 indicating that the mesoporous structure was retained after the loading process. However, 


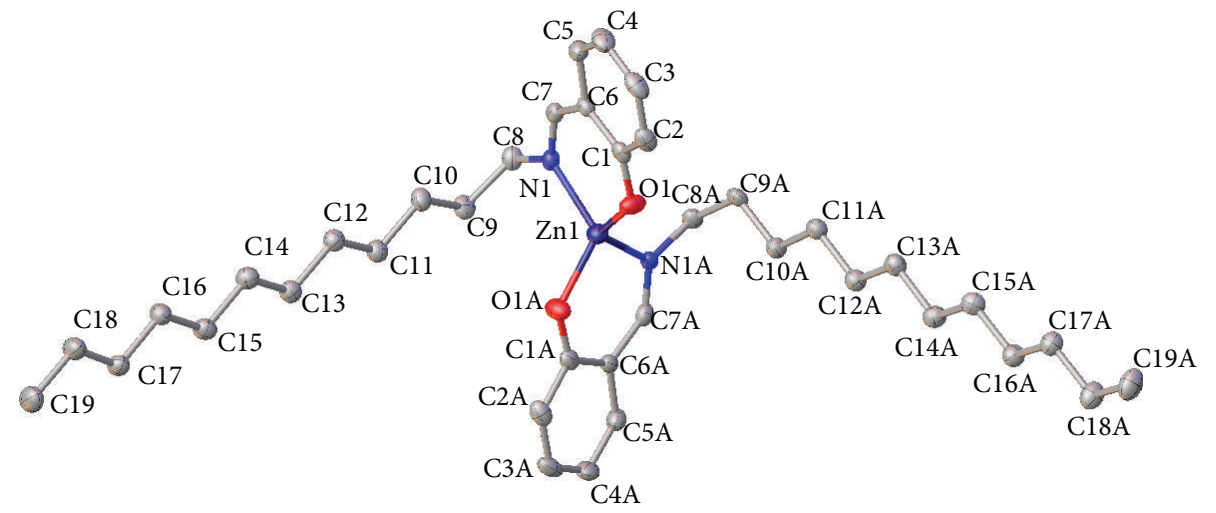

Figure 2: Molecular structure diagram of $\mathbf{1}$. Hydrogen atoms are omitted for clarity.

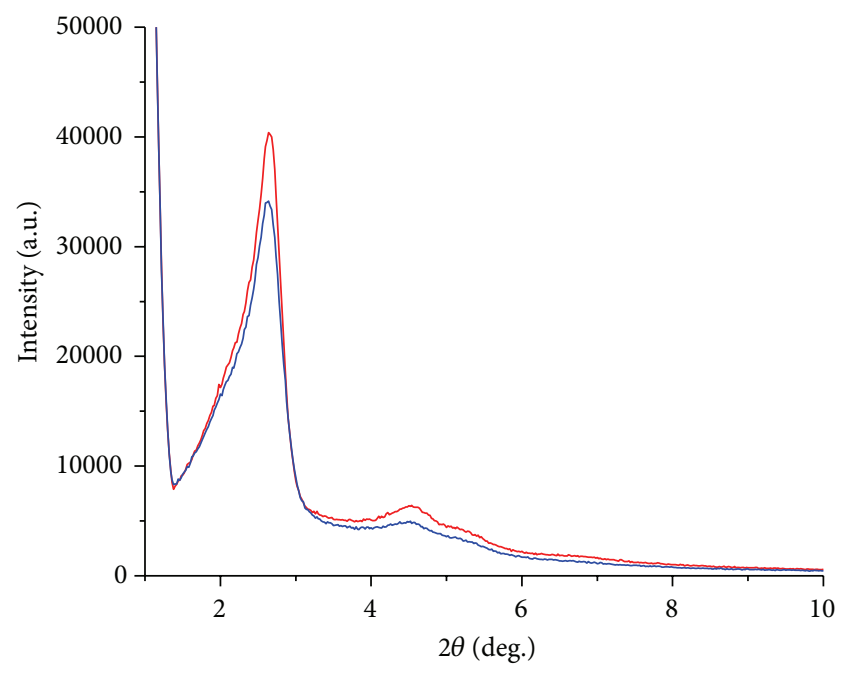

FIGURE 3: PXRD patterns of pure MCM-41 (red line) and MCM-41 loaded with 1 (blue line).

a decrease of the Bragg peak intensities is also observed, which could be due to the dispersion of $\mathbf{1}$ in the channels of MCM-41. The loading content of $\mathbf{1}$ was found to be $2.6 \%$ as determined by the XRF analysis.

Figure 4(a) shows the absorption spectra of $\mathbf{1}$ in ethanol solution $\left(1.0 \times 10^{-5} \mathrm{~mol} \mathrm{~L}^{-1}\right)$ and the solid host. It may be first noted that the $\mathrm{Zn}$ atom is not involved in these absorption spectra, since its $4 \mathrm{~s}$ orbital energy level is too high. Three major peaks can be unambiguously identified in the absorption spectrum of $\mathbf{1}$ in ethanol solution, which centered at around 361, 271, and $240 \mathrm{~nm}$, respectively. The wavelength below ca. $225 \mathrm{~nm}$ began to reach the solvent cut-off wavelength of ethanol. Thus, the absorption spectrum of 1 cannot be reliably recorded in this region. According to Kasha's rule [23], the $361 \mathrm{~nm}$ absorption peak can be appropriately assigned to the spatially allowed $l \rightarrow a_{\pi}$ transition of 1 , which corresponds to the excitation of the electrons from the lonepair orbitals of the oxygen atom to an antibonding orbital of $\pi$ origin $\left(a_{\pi}\right)$ of the aromatic ring. The $\pi \rightarrow \pi^{*}$ transitions of the aromatic ring of 1 should account for the 271 and $240 \mathrm{~nm}$ absorption peaks.

The absorption bands of $\mathbf{1}$ in the solid host show substantial broadening compared to those of $\mathbf{1}$ in ethanol solution. An obvious shoulder peak plus three major peaks can be clearly identified in the absorption spectrum of $\mathbf{1}$ in the solid host. The shoulder peak located at $c a .393 \mathrm{~nm}$, while the rest three peaks centered at around 330, 256, and $211 \mathrm{~nm}$, respectively. The nature of these absorption peaks could be also due to the $l \rightarrow a_{\pi}$ and $\pi \rightarrow \pi^{*}$ transitions of 1 . What sparked our interest is the observation of a large red shift $(\sim 40 \mathrm{~nm})$ of the absorption onset (and also considering the uprising part immediately followed by the absorption onset) as highlighted in the inset of Figure 4(a), when the microenvironment of 1 changes from the solvent ethanol to the inorganic solid medium. The shift of the absorption onset reflects the variation of the electronic energy levels of $\mathbf{1}$. Employing the concept of the Hückel molecular orbital (HMO) theory, a marked red-shift of the absorption onset can be associated with a band-gap reduction between the highest occupied molecular orbital (HOMO) and the lowest unoccupied molecular orbital (LUMO). Given that $\mathbf{1}$ can be treated as dispersive molecules in both of the above media and tends to optimize its Van der Waals interactions with its surroundings in the solid host, these interactions are amplified by the surface curvature of the pore walls, which interact with $\mathbf{1}$. Thus, the reduction of the HOMO-LUMO band gap can be reasonably attributed to the confined space effect.

Figure 4(b) shows the fluorescence emission spectra of 1 in ethanol solution $\left(1.0 \times 10^{-5} \mathrm{~mol} \mathrm{~L}^{-1}\right)$ and the solid host. Excited by $355 \mathrm{~nm}$ UV light, the emission peaks of $\mathbf{1}$ in both media can be reasonably assigned to the fluorescence from the intraligand excited state. A $5 \mathrm{~nm}$ red shift of the emission peaks from $466 \mathrm{~nm}$ of 1 in ethanol solution to $471 \mathrm{~nm}$ of 1 in the solid host was observed. Although displaying the same red-shift trends, the shift of absorption onset $(\sim 40 \mathrm{~nm})$ is much more prominent than that of the emission peaks $(5 \mathrm{~nm})$. A potential explanation of this phenomenon could be correlated with the nature of the $l \rightarrow a_{\pi}$ transition, which depends on the twist angle of the axes of the lone-pair orbitals relative to that of the adjacent $2 p_{\pi}$ orbitals of the attached aromatic ring $[23,24]$. It is possible that the confined space 


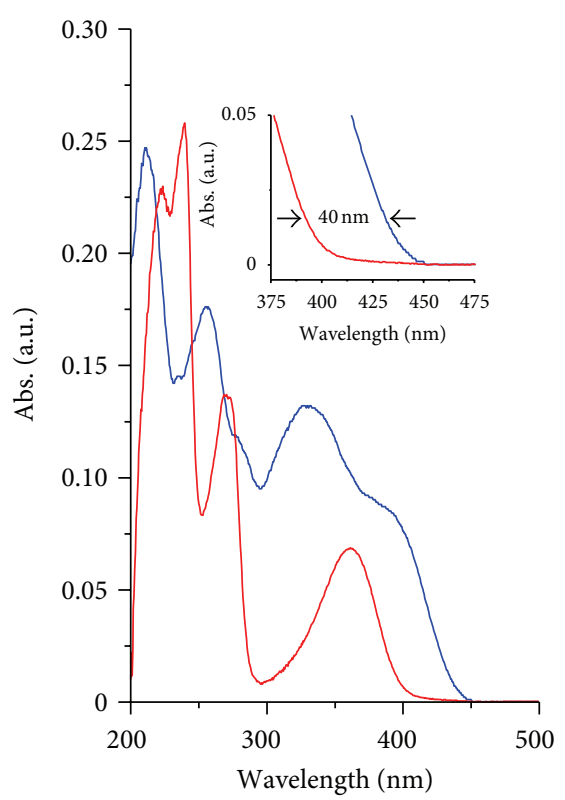

(a)

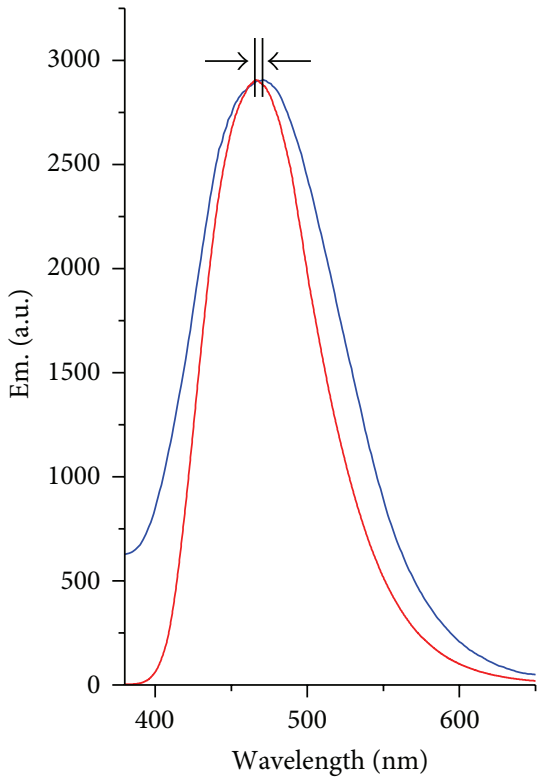

(b)

FIgURE 4: (a) Absorption spectra of 1 in ethanol solution (red line) and the solid host (blue line) at room temperature. The inset highlights the absorption onset of $\mathbf{1}$ in (a). (b) Fluorescence emission spectra of $\mathbf{1}$ in ethanol solution (red line) and the solid host (blue line) at room temperature. $\lambda_{\mathrm{ex}}=355 \mathrm{~nm}$.

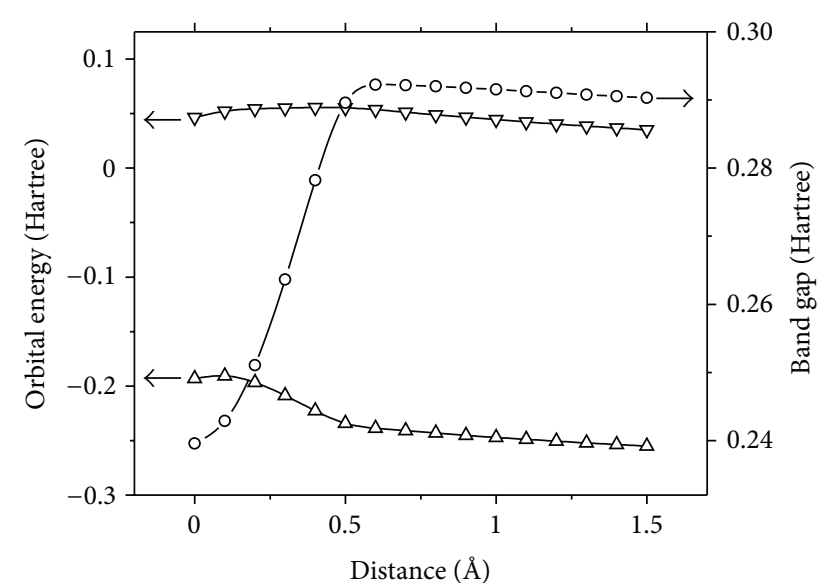

Figure 5: Plot of the energies of the HOMO $(\triangle)$, the LUMO $(\nabla)$, and the band gap $(\mathrm{O})$ of $\mathbf{1}$ at different $\mathbf{1}$-mica sheet distances. 1 Hartree $=$ $27.2 \mathrm{eV}$.

exerts some specific effect on the $l \rightarrow a_{\pi}$ transition by, for example, affecting the twist angle of the orbital axes, which is likely to result in the recorded change of the absorption spectra of $\mathbf{1}$. However, the molecular configuration of $\mathbf{1}$ could undergo transient alteration as a result of the light absorption, thus compromising the confined space effect. Consequently, a less distinctive red shift of the emission peaks was observed as shown in Figure 4.

With the aim of providing a preliminary quantum chemical interpretation of the above electronic property variation at a reasonable computational cost, semiempirical calculations at the PM3 level using the Gaussian 09 suite of programs were carried out. The atomic coordinates of 1 employed in the calculations were based on the X-ray crystallographic data of this work. Concerning that $\mathbf{1}$ is confined by the inner pore surface of the solid host, the microenvironment of $\mathbf{1}$ can be simulated by placing $\mathbf{1}$ parallel to a mica sheet (a layered silicate formed by two-dimensional arrays of $\mathrm{SiO}_{4}$ tetrahedra sharing the edge). Through a progressive increase of the distance between 1 and the mica sheet from 0.0 up to $1.5 \AA$, the energies of the HOMO, the LUMO, and the band gap of $\mathbf{1}$ are calculated and then plotted in Figure 5. Results revealed a remarkable increase of the band gap of 1 , when the 1-mica sheet distance increases from 0.0 to $0.6 \AA$. However, the variation in band gap generally levels off in the range of $0.6-1.5 \AA$. This indicates that the confined space effect is most likely distance dependent. It is evident from Figure 5 that the key factor contributing to the increase of the band gap in the range of $0.0-0.6 \AA$ is the decrease of the HOMO energy. The variation of the LUMO energy in this range is less significant compared to that of the HOMO energy. This implies that within an effective distance range, the influence of the confined space effect on molecular orbitals is different: the HOMO is more sensitive than the LUMO.

To offer a pictorial way of illustrating the confined space effect, extended HMO calculations were performed on this 1-mica sheet system. The atomic coordinates used in the extended HMO calculations are exactly the same as those used in the above semiempirical calculations. Figure 6 shows the contour plots of the HOMO surfaces for the 1-mica sheet system separated by 0.0 and $0.6 \AA$, respectively. It can be seen from the figure that when $\mathbf{1}$ approaches the mica surface from 0.6 to $0.0 \AA$, the electron density in $\mathbf{1}$ becomes significantly 


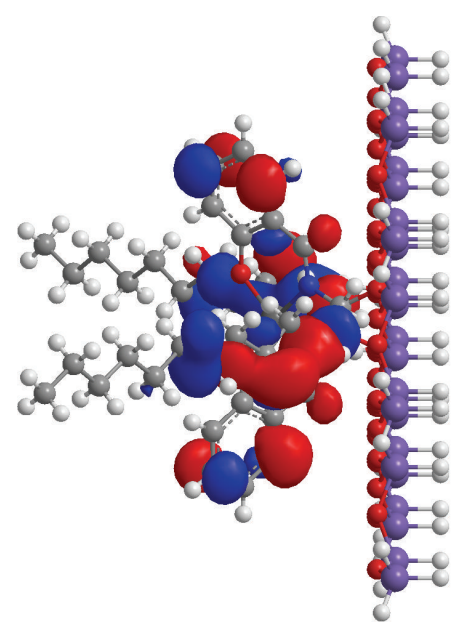

(a)

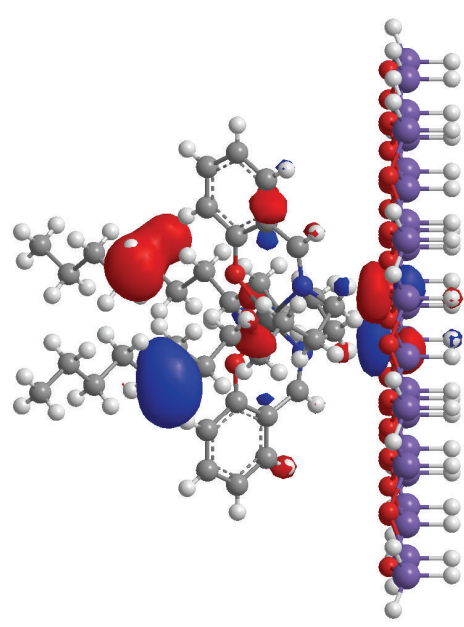

(b)

FIGURE 6: Contour plots of the HOMO surfaces for the 1-mica sheet system. (a) 1-mica sheet distance: 0.0 Å. (b) 1-mica sheet distance: $0.6 \AA$. Positive and negative values of the orbital contour are displayed in red ( 0.01 a.u.) and blue ( -0.01 a.u. $)$, respectively.

higher. We postulate that the enhanced electron density is a direct reflection of the confined space effect.

Before ending this paper, we would like to mention the recently published themed issue on "Molecules in Confined Spaces" in PCCP [11]. These beautiful works showcase that the study of molecular confinement is actively involved in many fields of chemical research, especially host-guest chemistry and heterogeneous catalysis. The investigation of the tendencies in the electronic structures, energy spectra, and chemical reactivity of spatially confined atoms and molecules has been of particular interest. It is noteworthy that whether or not the decrease of the HOMO-LUMO band gap for confined guest molecules can be exclusively assigned to the electronic confinement is still a matter of debate. Scientists from Spain performed semiempirical electronic structure calculations on confined anthracene and DFT/Hartree-Fock calculations on confined naphthalene $[25,26]$. They concluded that the confined space effect led to the reduction of the HOMO-LUMO band gap, consistent with the spectroscopically observed red shifts in the $0-0$ optical transitions. On the other hand, the computation results from a team of Belgian and English scientists gave an increase in the HOMO-LUMO band gap [27]. To gain further insight into this matter, a thorough analysis of the influence of the confinement on the excitation energies of anthracene and naphthalene based on time-dependent DFT was carried out [28]. On the basis of their results, it was presumed that the decrease in the excitation energies derived from the above spectroscopic observations should not be exclusively linked to the compression of the molecular orbitals. Obviously, a future effort of combined experimental and theoretical investigations is still needed to achieve a more comprehensive understanding of the confined space effect.

\section{Conclusions}

In summary, a new zinc(II)-Schiff base complex (1) was synthesized and subsequently loaded in an inorganic solid host in this work. A large red shift $(\sim 40 \mathrm{~nm})$ of the absorption onset was recorded, when the microenvironment of 1 changed from the solvent ethanol to the inorganic solid medium, evidencing the confined space effect. The marked shift of the absorption onset was associated with a band-gap reduction between the HOMO and the LUMO. Theoretical calculation results showed that the confined space effect is distance dependent and exerts a more profound influence on the HOMO than the LUMO within an effective distance range. An initial study implied that the confined space effect is also accompanied with the electron density variation. Our future work intends to see if the confined space effect could bring forth its usefulness in the study of biological systems.

\section{Acknowledgments}

This work was supported by the Open Project of MOE Key Laboratory of Optical Information Science \& Technology (no. 2012KFKT-03), the Fundamental Research Funds for the Central Universities, and the National Innovation Training Program for University Students (no. 201310055011). The authors wish to thank their colleagues at Nankai University for their assistance in X-ray crystallography and computational works.

\section{References}

[1] N. Kielland, E. C. Escudero-Adan, M. M. Belmonte, and A. W. Kleij, "Unsymmetrical octanuclear Schiff base clusters: synthesis, characterization and catalysis," Dalton Transactions, vol. 42, no. 5, pp. 1427-1436, 2013.

[2] J. Zuo, C. Bi, Y. Fan et al., "Cellular and computational studies of proteasome inhibition and apoptosis induction in human cancer cells by amino acid Schiff base-copper complexes," Journal of Inorganic Biochemistry, vol. 118, pp. 83-93, 2013.

[3] J. Jankowska, M. F. Rode, J. Sadlej, and A. L. Sobolewski, "Photophysics of Schiff bases: theoretical study of salicylidene methylamine," ChemPhysChem, vol. 13, no. 18, pp. 4287-4294, 2012. 
[4] M. Ganguly, A. Pal, Y. Negishi, and T. Pal, "Diiminic Schiff bases: an intriguing class of compounds for a coppernanoparticle-induced fluorescence study," Chemistry - A European Journal, vol. 18, no. 49, pp. 15845-15855, 2012.

[5] J. W. Leeland, F. J. White, and J. B. Love, "Encapsulation of a magnesium hydroxide cubane by a bowl-shaped polypyrrolic schiff base macrocycle," Journal of the American Chemical Society, vol. 133, no. 19, pp. 7320-7323, 2011.

[6] A. B. Canaj, D. I. Tzimopoulos, A. Philippidis, G. E. Kostakis, and C. J. Milios, "Employment of a new tripodal ligand for the synthesis of cobalt(II/III), nickel(II), and copper(II) clusters: magnetic, optical, and thermal properties," Inorganic Chemistry, vol. 51, no. 19, pp. 10461-10470, 2012.

[7] A. K. Yadava, H. S. Yadav, S. Singh, U. S. Yadav, and D. P. Rao, "Synthesis and characterization of some novel Schiff base complexes of oxovanadium(IV) cation," Journal of Chemistry, vol. 2013, Article ID 689518, 5 pages, 2013.

[8] A. A. El-Bindary, A. Z. El-Sonbati, M. A. Diab, and M. K. Abd El-Kader, "Potentiometric and thermodynamic studies of some Schiff-base derivatives of 4-aminoantipyrine and their metal complexes," Journal of Chemistry, vol. 2013, Article ID 682186, 6 pages, 2013.

[9] J. Safari, Z. Zarnegar, and F. Rahimi, "An efficient oxidation of benzoins to benzils by manganese(II) Schiff base complexes using green oxidant," Journal of Chemistry, vol. 2013, Article ID 765376, 7 pages, 2013.

[10] J. S. Kumaran, S. Priya, N. Jayachandramani, and S. Mahalakshmi, "Synthesis, spectroscopic characterization and biological activities of transition metal complexes derived from a tridentate Schiff base," Journal of Chemistry, vol. 2013, Article ID 260358, 10 pages, 2013.

[11] R. A. Schoonheydt and B. M. Weckhuysen, "Editorial highlight: molecules in confined spaces," Physical Chemistry Chemical Physics, vol. 11, no. 16, pp. 2794-2798, 2009.

[12] C. G. Wu and T. Bein, "Conducting polyaniline filaments in a mesoporous channel host," Science, vol. 264, no. 5166, pp. 17571759, 1994.

[13] S. Zhu, J. Chen, H. Li, and Y. Cao, "Structure and conformation of poly(ethylene glycol) in confined space of montmorillonite," Applied Surface Science, vol. 264, pp. 500-506, 2013.

[14] Y. Liu and B. Chakraborty, "Segregation of polymers in confined spaces," Physical Biology, vol. 9, no. 6, Article ID 066005, 2012.

[15] X. F. Wang and Q. L. Yuan, "Preparation of polystyrene nanospheres in confined space," Acta Chimica Sinica, vol. 70, no. 9, pp. 1047-1054, 2012.

[16] Y. Kitayama, S. Tomoeda, and M. Okubo, "Experimental evidence and beneficial use of confined space effect in nitroxidemediated radical microemulsion polymerization (microemulsion NMP) of n-butyl acrylate," Macromolecules, vol. 45, no. 19, pp. 7884-7889, 2012.

[17] C. G. Yu and J. He, "Synergic catalytic effects in confined spaces," Chemical Communications, vol. 48, no. 41, pp. 4933-4940, 2012.

[18] K. K. Bania, D. Bharali, B. Viswanathan, and R. C. Deka, "Enhanced catalytic activity of zeolite encapsulated Fe(III)Schiff-base complexes for oxidative coupling of 2-napthol," Inorganic Chemistry, vol. 51, no. 3, pp. 1657-1674, 2012.

[19] M. Bagherzadeh and M. Zare, "Synthesis and characterization of NaY zeolite-encapsulated Mn-hydrazone Schiff base: an efficient and reusable catalyst for oxidation of olefins," Journal of Coordination Chemistry, vol. 65, no. 22, pp. 4054-4066, 2012.
[20] A. Moghimi and M. J. Poursharifi, "Solid phase extraction of mercury(II) from natural water by octadecyl-bonded silicaloaded schiff's base 4,4'-dimethoxybenzil bisthiosemicarbazone phases," Asian Journal of Chemistry, vol. 23, no. 6, pp. 25312535, 2011.

[21] C. M. Kowalchuk, G. Schmid, W. Meyer-Zaika, Y. Huang, and J. F. Corrigan, "Preparation, characterization, and condensation of copper tellurolate clusters in the pores of periodic mesoporous silica MCM-41," Inorganic Chemistry, vol. 43, no. 1, pp. 173-180, 2004.

[22] Y.-Q. Huo, S.-W. Wang, H.-L. Cui, L. Tang, J. Li, and F.$\mathrm{X}$. Zhang, "Synthesis, crystal structures and fluorescence of $\mathrm{Zn}$ (II) complexes with schiff base," Chinese Journal of Inorganic Chemistry, vol. 26, no. 7, pp. 1274-1278, 2010.

[23] M. Kasha and H. R. Rawls, "Correlation of orbital classification of molecular electronic transitions with transition mechanism: the aromatic amines," Photochemistry and Photobiology, vol. 7, no. 6, pp. 561-569, 1968.

[24] E. C. Lim and S. K. Chakrabarti, "Role of $l \rightarrow a_{\pi}$ transitions in spin-orbit coupling of aromatic amines: phosphorescence of aniline and its $N$-alkyl derivatives," The Journal of Chemical Physics, vol. 47, no. 11, pp. 4726-4730, 1967.

[25] F. Márquez, H. García, E. Palomares, L. Fernández, and A. Corma, "Spectroscopic evidence in support of the molecular orbital confinement concept: case of anthracene incorporated in zeolites," Journal of the American Chemical Society, vol. 122, no. 27, pp. 6520-6521, 2000.

[26] F. Márquez, C. M. Zicovich-Wilson, A. Corma, E. Palomares, and H. García, "Naphthalene included within all-silica zeolites: influence of the host on the naphthalene photophysics," Journal of Physical Chemistry B, vol. 105, no. 41, pp. 9973-9979, 2001.

[27] A. Borgoo, D. J. Tozer, P. Geerlings, and F. De Proft, "Influence of confinement on atomic and molecular reactivity indicators in DFT," Physical Chemistry Chemical Physics, vol. 10, no. 10, pp. 1406-1410, 2008.

[28] A. Borgoo, D. J. Tozer, P. Geerlings, and F. De Proft, "Confinement effects on excitation energies and regioselectivity as probed by the Fukui function and the molecular electrostatic potential," Physical Chemistry Chemical Physics, vol. 11, no. 16, pp. 2862-2868, 2009. 

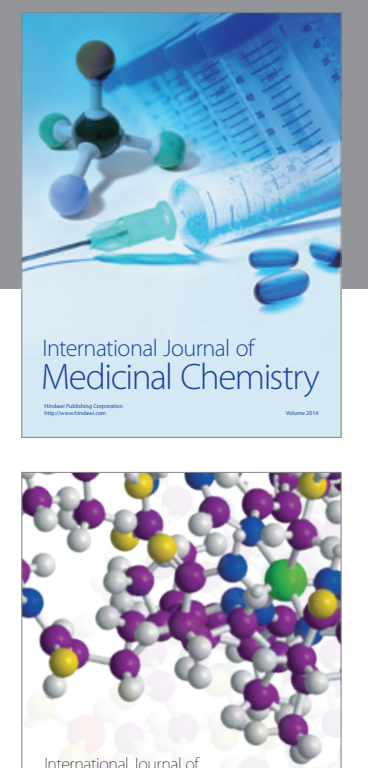

\section{Carbohydrate} Chemistry

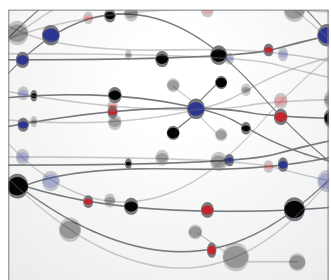

The Scientific World Journal
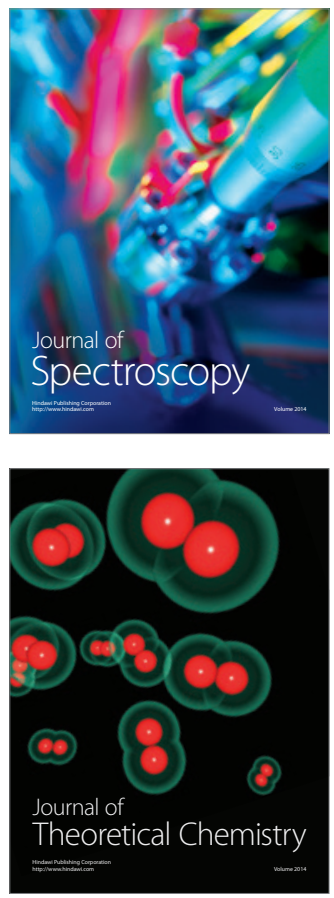
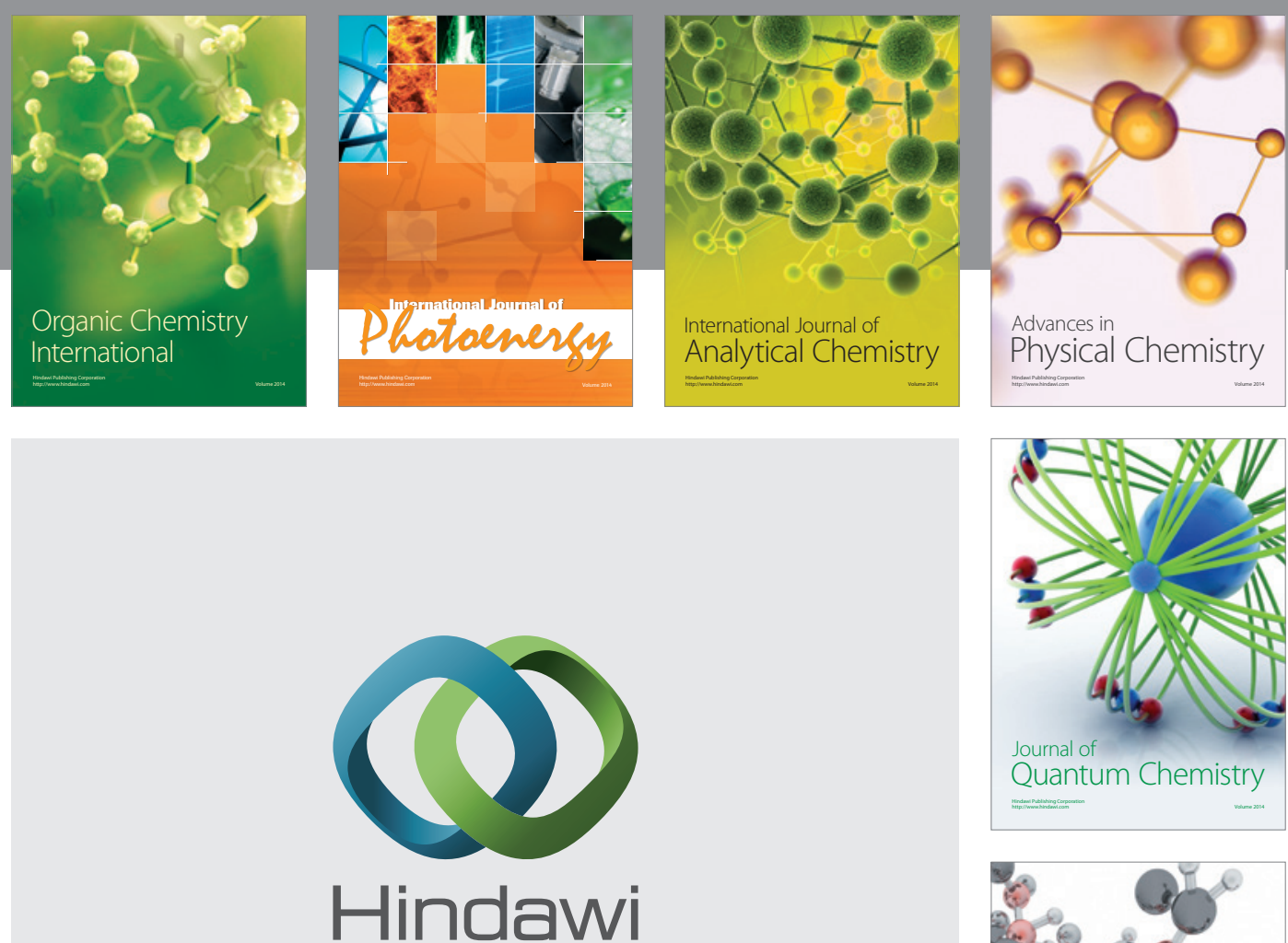

Submit your manuscripts at

http://www.hindawi.com

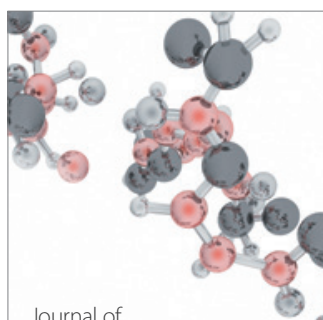

Analytical Methods

in Chemistry

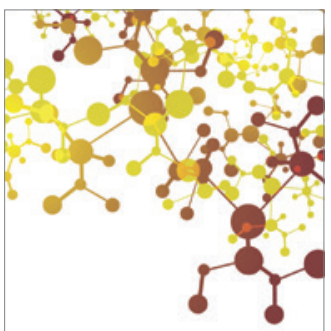

Journal of

Applied Chemistry

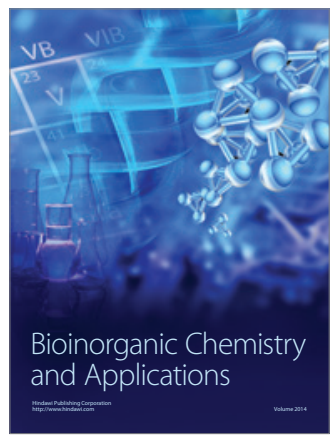

Inorganic Chemistry
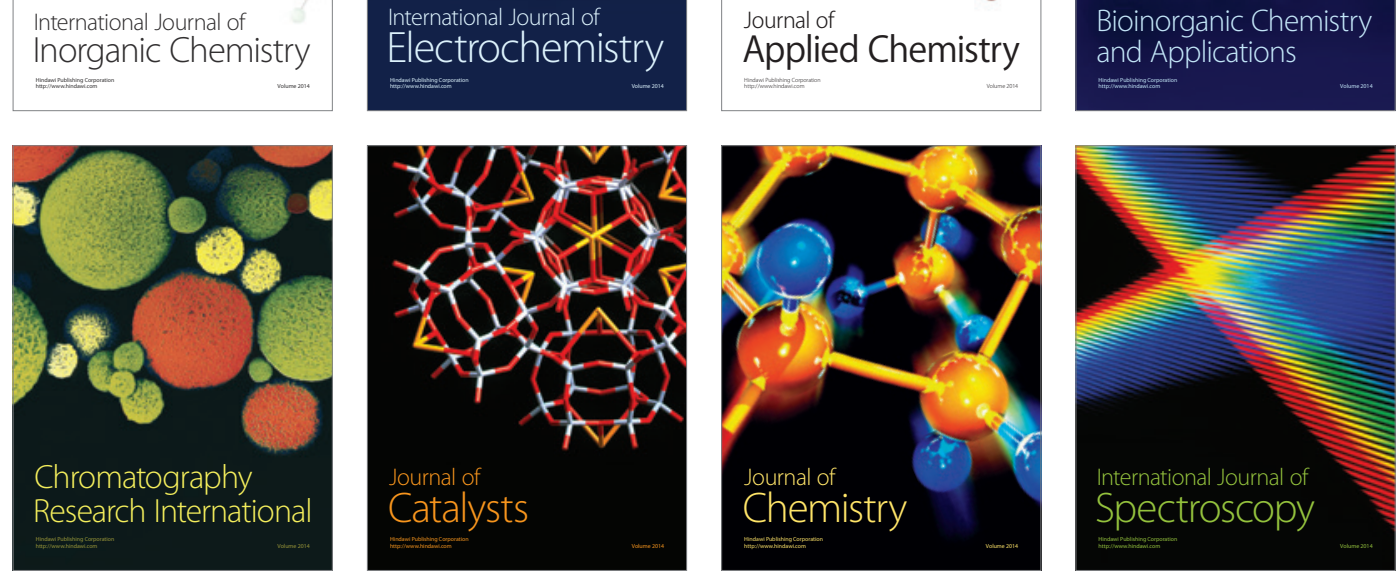